\section{Heteromodal Cortex}

Kerry Donnelly

VA WNY Healthcare System, University of Buffalo (SUNY) Behavioral Health Careline (116B), Buffalo, NY, USA

\section{Synonyms}

Heteromodal association cortex; Tertiary cortex

\section{Definition}

The heteromodal cortex refers to a region that receives input from multiple sensory or multimodal areas. Two major areas of heteromodal cortex are generally identified. Posteriorly, there is the PTO cortex (the confluence of the parietal, temporal, and occipital lobes), as well as certain occipitotemporal and occipitoparietal areas. Essentially, these are areas that receive input from multiple unimodal sensory association areas and/or other heteromodal cortices. Anteriorly, the prefrontal cortices are generally considered multimodal or heteromodal in that they also receive input from multiple sensory or other polymodal cortices. Because of their multimodal inputs, these cortical areas are considered to be responsible for more complex or integrated cognitive activities. For example, when we think of an apple, we imagine not only how it looks and tastes, but also its smell, its texture, its weight, and even how it sounds when we to bite into it. This "multimodal sensory image" is thought to be the result of the integration that takes place in the posterior heteromodal cortex. Similarly, the prefrontal association cortices utilize multiple sensory, limbic, and motor areas in the course of its supraordinate planning, organization, and ongoing monitoring of behavioral responses to the environment. Lesions in the PTO may result in impaired recognition of faces, objects, or voices. Anterior heteromodal lesions are more likely to result in a "frontal lobe syndrome." This syndrome is typified by deficient attention and executive functions, along with behavioral changes. The behavioral abnormalities often follow one of two paths: apathy, with attendant loss of initiative and emotional blunting, or disinhibition, marked by impulsivity and poor judgment and insight.

\section{References and Readings}

Mendoza, J. E., \& Foundas, A. L. (2008). Clinical neuroanatomy: A neurobehavioral approach (pp. 313-314, 387-390, 393-395, 403-405, 409-455). New York: Springer.

Mesulam, M. (2000a). Chapter 1. Large-scale networks, association cortex, frontal syndromes, the limbic system, and hemispheric specialization. In M. Mesulam (Ed.), Principles of behavioral neurology (pp. 1-120). Philadelphia: F. A. Davis.

Mesulam, M. M. (2000b). Principles of behavioral and cognitive neurology (2nd ed.pp. 34-36., 41-48). New York: Oxford. 